\title{
Preditor de qualidade de vida em indivíduos com doença de Parkinson moderada
}

https://doi.org/10.11606/issn.1981-4690.v35i1p15-24

\section{Resumo}

0 objetivo deste estudo foi verificar dentre os sintomas motores, sintomas não motores e o medo de cair quais seriam os principais preditores de qualidade de vida em indivíduos nos estágios 2 e 3 da doença de Parkinson (DP). Trinta e nove indivíduos com DP $(64,1 \pm 9,1$ anos, $10,3 \pm 4,7$ duração da DP) avaliados no estado on da medicação participaram do estudo. A disfunção motora foi avaliada por meio dos seguintes testes: parte III da Escala Unificada de Avaliação da Doença de Parkinson (UPDRS-III), teste Timed up and Go (TUG), Teste de Sistema de Avaliação do Equilibrio (BESTest) e uma repetição máxima dos membros inferiores (1RM no leg press). A disfunção não motora foi avaliada por meio dos seguintes testes: Avaliação Cognitiva de Montreal (MoCA), Inventário de Depressão de Beck (IDB) e o Índice de Qualidade do Sono de Pittsburgh (PSOI). 0 medo de cair foi avaliado por meio da Escala Internacional de Eficácia de Quedas (FES-I). A qualidade de vida foi avaliada por meio do teste Questionário da Doença de Parkinson (PDQ-39). Uma regressão múltipla linear, método stepwise foi empregada para verificar o principal preditor do escore da PDQ-39. A única variável independente que entrou no modelo de regressão múltipla linear (stepwise) e mostrou uma alta capacidade para explicar o escore de qualidade de vida de indivíduos com DP foi a FES-I (R2 ajustado $=0,73, P<0,0001$ ). Assim, como implicação clínica, é possivel sugerir que estratégias de treinamento físico que promovam diminuição no medo de cair podem impactar positivamente na qualidade de vida de indivíduos com DP moderada.

PalavRas-chave: Medo de cair; Sintomas motores; Instabilidade postural; Atividades da vida diária.

\section{Introdução}

A doença de Parkinson (DP) é uma patologia neurodegenerativa progressiva e sua principal causa é a disfunção dos núcleos da base que ocorre pela degeneração de neurônios na via nigroestriatal dopaminérgica ${ }^{1}$. A degeneração dessas estruturas resulta em uma variedade de disfunçóes que afetam negativamente a qualidade de vida de indivíduos com a DP. Por exemplo, vários estudos apontaram que as disfunções motoras, tais como mobilidade reduzida, sintomas motores (i.e., rigidez muscular, bradicinesia, tremor de repouso, instabilidade postural), déficit de força e de equilíbrio, seriam os principais preditores de qualidade de vida em indivíduos com a DP ${ }^{2-5}$. Enquanto outros têm demonstrado que as disfunções não motoras da DP como comprometimento cognitivo, distúrbios do sono e depressão, podem ser os principais preditores de qualidade de vida nessa populaçãoo $0^{5-8}$. Por outro lado, o medo de cair é um relato comum em indivíduos com DP e cerca de $70 \%$ dos indivíduos relatam perda de autonomia e limitaçóes das atividades devido ao medo de cair $^{9-11}$. Apesar do crescente número de estudos que analisaram o impacto das disfunçóes motoras, não motoras e medo de cair na qualidade de vida dessa população, pouco se conhece sobre os principais fatores que podem predizer a qualidade de vida em indivíduos no estágio moderado da DP.

Estudos de associaçáo tem utilizado indivíduos com estágios variados da $\mathrm{DP}^{4,12-14}$ o que pode 
comprometer a interpretação dos resultados. Por exemplo, indivíduos em estágio inicial da DP não apresentam comprometimento axial, já os indivíduos em estágios moderados apresentam comprometimento axial com alguma instabilidade postural ${ }^{15}$. Sabe-se que a instabilidade postural representa um grande desafio terapêutico, uma vez que o tratamento farmacológico e a deep brain stimulation tem efeito limitado sobre ela ${ }^{16-18}$. Neste sentido, é possível hipotetizar que em indivíduos com moderada DP, a instabilidade postural poderia ser o principal sintoma motor preditor de qualidade de vida pobre. Adicionalmente, sabe-se que o medo de cair é influenciado pela instabilidade postural apresentada por indivíduos

\section{Método}

\section{Desenho do Estudo}

Este foi um estudo transversal onde as disfunçóes motoras, não motoras e o medo de cair foram tratadas como variáveis independentes e a qualidade de vida foi tratada como variável dependente.

\section{Amostra}

Trinta e nove indivíduos com DP participaram do estudo e foram recrutados da Associação Brasil Parkinson. O diagnóstico clínico de idiopática DP foi confirmado por um neurologista de acordo com os critérios clínicos do Banco de Cérebro de Londres (UK Parkinson's disease Society Brain Bank Diagnostic Criteria ${ }^{19}$. Os critérios de inclusão foram: 1 ) gravidade da DP entre os estágios 2 e 3 de acordo com a escala modificada de Hoehn-Yahr ${ }^{20}$; 2) idade entre 50 e 80 anos; 3) estabilidade no uso da medicaçáo; 4) nível de cogniçáo com pontuaçáo maior que 23 de acordo com o Mini Exame do Estado Mental ${ }^{21}$; 5) nível de escolaridade maior que 4 anos; 6) não ter participado de qualquer treinamento físico nos últimos 3 anos (indivíduos treinados ; 7) nenhuma outra desordem neurológica, além da DP; 8) nenhuma doença cardiovascular, aneurisma, artrite significante e alteraçōes visuais graves. É importante ressaltar que nâo foram incluídos no estudo indivíduos no estágio inicial (1 na escala de Hoehn-Yahr) ou muito grave da DP (4 e 5 na escala de Hoehn-Yahr) devido à grande variabilidade intra-indivíduos reportada nos estudos com moderada DP9. Assim, a segunda hipótese deste estudo é que o medo de cair poderá ser o preditor multifatorial de qualidade de vida pobre em indivíduos com moderada DP.

Portanto, o objetivo desse estudo foi verificar dentre as disfunçóes motoras (i.e., sintomas motores, mobilidade, equilíbrio e força muscular), não motoras (i.e., comprometimento cognitivo, depressão e medo de cair) e o medo de cair quais seriam as principais preditores de qualidade de vida pobre em indivíduos no estágio moderado da DP. Estes achados poderiam ser de grande valia no intuito de aumentar a eficácia da utilização de estratégias de intervenções não farmacológicas (i.e., treinamento físico) para melhorar a qualidade de vida de indivíduos com DP moderada.

quando utilizado indivíduos em diferentes estágios da doença ${ }^{4,12-14}$. Adicionalmente, apenas indivíduos sem experiência em intervençóes de treinamento (longo prazo) foram incluídos no estudo uma vez que a prática de exercício físico melhora a qualidade de vida desses indivíduos ${ }^{22-24}$ o que prejudicaria o propósito do presente estudo. As características demográficas, antropométricas e clínicas dos indivíduos estão apresentadas na TABELA 1. Após serem informados sobre os objetivos, os benefícios e os riscos associados à execução do protocolo experimental, todos os indivíduos assinaram um termo de consentimento livre e esclarecido. Este estudo foi aprovado pelo Comitê de Ética da Escola de Educação Física e Esporte da Universidade de São Paulo (EEFE-USP) (número 2011/12) e registrado no Registro Brasileiros de Ensaios Clínicos (RBR-53S3RK).

\section{Procedimentos}

As disfunções motoras, não motoras e o medo de cair dos indivíduos foram avaliadas em cinco visitas ao Centro de Estudos em Psicobiologia do Exercício. Todas as avaliaçốes foram realizadas de 1,5 a 2 horas depois dos indivíduos com DP terem ingerido a sua dose dos medicamentos pela manhã (fase on da medicação). $\mathrm{Na}$ primeira visita foram avaliados os sintomas motores (parte III da Escala Unificada de Avaliação da Doença de Parkinson - UPDRS-III), o comprometimento cognitivo (Avaliaçáo Cognitiva de Montreal - MoCA), a depressão (Inventário de Depressão de Beck - IDB) 
e a qualidade de vida (Questionário da Doença de Parkinson - PDQ-39). Na segunda visita foram avaliados a qualidade do sono (Índice de Qualidade do Sono de Pittsburgh) mobilidade (timed up and go TUG), o medo de cair (Escala Internacional de Eficácia de Quedas - FES-I) e o equilíbrio (Teste de Sistema de Avaliação do Equilíbrio - BESTest). Na terceira, quarta e quinta visitas, com intervalo de 48 horas entre elas foi avaliada a força muscular dos membros inferiores por meio do teste de uma repetição máxima (1RM) no exercício leg-press.

\section{Avaliaçáo da qualidade de vida}

O PDQ-39 é umas das principais escalas utilizadas na literatura para avaliação da qualidade de vida da $\mathrm{DP}^{25}$. O PDQ-39 apresenta um total de 39 questóes e a resposta de cada questáo varia de 0 (nunca) a 4 (sempre). As questóes são referidas a que frequência o indivíduo tem passado dificuldades decorrentes da DP durante o último mês. Esse questionário contém oito domínios: 1) mobilidade (10 questóes); 2) atividades de vida diária (6 questôes); 3) estado emocional (6 questóes); 4) estigma social (4 questóes); 5) apoio social (3 questóes); 6) cogniçãao (4 questóes); 7) comunicação (3 questóes); e 8) desconforto físico (3 questóes). O escore total para cada indivíduo é calculado de acordo com a seguinte fórmula: $100 \mathrm{x}$ soma dos escores do indivíduo nas 39 questóes / 4 x 39). A pontuação total no PDQ-39 varia de 0 (nenhum problema) a 100 (máximo nível de problema), ou seja, uma baixa pontuação indica melhor percepção da qualidade de vida por parte do indivíduo ${ }^{25}$. O escore da PDQ-39 foi utilizado para análise estatística.

\section{Avaliação das disfunçóes motoras}

A UPDRS-III é a principal escala para avaliação da gravidade motora da $\mathrm{DP}^{26}$ que inclui 14 itens começando no item número 18 e terminando no item número 31. Cada item alcança de 0 a 4 (0 nenhum sintoma e 4 sintomas graves). A maioria desses 14 itens avalia os membros superiores e inferiores, direito e esquerdo podendo chegar ao escore máximo de 108 pontos, o que indica grave comprometimento motor ${ }^{26}$.

OTUG foi relatado como uma ferramenta sensível e confiável para avaliar a mobilidade de indivíduos com $\mathrm{DP}^{27}$. Este teste foi aplicado da seguinte forma: o indivíduo iniciava o teste sentado em uma cadeira de $46 \mathrm{~cm}$ de altura, com os braços estendidos sobre as coxas e os pés totalmente apoiados no solo. Ao sinal do avaliador, o indivíduo levantava, sem o auxílio das mãos, e caminhava o mais rápido possível, sem correr, contornava um cone posicionado a uma distância de três metros da cadeira e retornava à posição inicial. $\mathrm{O}$ cronômetro era acionado a partir do sinal do avaliador e parado quando o indivíduo sentava totalmente na cadeira ${ }^{28}$. Após a demonstração do avaliador, o indivíduo fazia duas tentativas do teste, separadas por um minuto de intervalo, para familiarizar-se com os procedimentos. A seguir, o indivíduo realizava duas tentativas do teste propriamente dito, com um minuto de intervalo entre elas. O melhor escore (menor tempo em segundos) foi usado para a análise estatística.

Como a instabilidade postural em indivíduos com DP é multifatorial ${ }^{29}$, todos os déficits causados por ela podem ser avaliados de uma só vez por meio do BESTest $^{30}$. O BESTest contém 36 itens agrupados em seis sistemas: I) limitaçóes biomecânicas, (II) limite de estabilidade/verticalidade, (III) ajustes posturais antecipatórios, (IV) respostas posturais, (V) orientação sensorial, e (VI) estabilidade na marcha. Cada sistema é pontuado de 0 a 3. Uma pontuação de 0 indica que a pessoa é incapaz de desempenhar a tarefa enquanto que uma pontuaçáo de 3 indica capacidade adequada de realização da mesma. Assim, quanto maior o escore melhor o equilíbrio do indivíduo. $\mathrm{O}$ escore máximo do BESTest é uma porcentagem derivada da divisão do atual escore pelo possível total escore de 108 e multiplicada por 100. O escore de cada sistema também é reportado como porcentagem, derivada da divisão do atual escore pelo total possível escore do sistema e multiplicada por $100^{30}$. O escore do BESTest e o escore de cada sistema foram usados para análise estatística.

O teste de 1RM dos membros inferiores foi avaliado através do exercício leg-press $90^{\circ}$, de acordo com os procedimentos da American Society of Exercise Physiologists $^{31}$. Os indivíduos realizaram duas sessóes de familiarização com os procedimentos do teste para estabilização dos valores de 1RM e variabilidade entre as sessóes menor que 5\% e, posteriormente, realizaram o teste propriamente dito. As sessóes de familiarização e o teste foram separados por no mínimo 48 horas. Previamente a cada sessão de teste, os indivíduos realizaram um aquecimento no ciclo ergômetro entre 50 e 60 rpm, durante 5 minutos. Após o aquecimento geral, os indivíduos executaram um aquecimento específico composto por uma série de cinco repetiçóes com uma carga de aproximadamente 50\% de 1RM ou do peso corporal do indivíduo e uma série de três repetiçóes com uma carga de aproximadamente $70 \%$ de 1RM estimada ou do peso corporal do indivíduo, com dois minutos de intervalo entre as séries. Após o aquecimento, foi iniciado o teste de 1RM. Os indivíduos 
tiveram cinco tentativas, com três minutos de intervalo entre elas, para a obtenção da força dinâmica máxima. Os incrementos de peso $(\mathrm{kg})$ em cada tentativa variaram conforme a percepção de esforço do indivíduo e pela experiência do pesquisador na aplicação do teste. Foi considerado para análise estatística a maior carga de $1 \mathrm{RM}$ na qual o indivíduo conseguiu levantar em um ciclo completo do movimento no exercício leg-press $90^{\circ}$.

\section{Avaliação das disfunçóes não motoras}

O comprometimento cognitivo foi avaliado através da MoCA que foi desenhada como um instrumento de rastreio para comprometimento cognitivo leve ${ }^{32}$. A MoCA foi conduzida em um quarto quieto sem distraçóes. $\mathrm{O}$ escore máximo de 30 pontos indica um ótimo desempenho cognitivo e um escore $\leq 25$ pontos indicam comprometimento cognitivo leve $\mathrm{e}^{32}$. Um ponto é adicionado ao escore total para indivíduos que têm escolaridade menor que 12 anos. AMoCA avalia 7 domínios cognitivos: visuo-espacial e função executiva (5 pontos), nomeação (3 pontos), atençáo (6 pontos), linguagem (3 pontos), abstraçáo (2 pontos), memória (5 pontos) e orientação (6 pontos). O escore da MoCA foi usado para análise estatística.

O IDB apresenta boas propriedades psicométricas e tem sido largamente utilizado para análise da depressão em indivíduos com $\mathrm{DP}^{33}$. O teste é composto por um questionário que contem 21 itens, que analisam domínios específicos (tristeza, pessimismo, sensação de fracasso, perda de satisfação, sentimentos de punição, sentimentos de culpa, autodepreciação, autoacusação, ideais suicidas, irritabilidade, crises de choro, indecisão, retração social, distorção da imagem corporal e perda de peso) e não específicos (inibição para o trabalho, distúrbios de sono, fadiga, perda de apetite, preocupação e diminuição da libido) e variam em opçóes de respostas de $0-3$, com um escore máximo de $63^{34}$. O escore do IDB foi usado para análise estatística.

O PSQI é um entre os seis questionários recomendados pela força tarefa da Sociedade de Distúrbios do Movimento para avaliar problemas gerais do sono e medir a gravidade dos problemas de sono de pacientes com DP ${ }^{35}$. O PSQI interpreta a percepção da qualidade do sono com uma alta reprodutibilidade teste-reteste (Cronbach's alpha> $0,80)^{36}$. O escore PSQI global é composto por sete componentes: qualidade subjetiva do sono, latência do sono, duração do sono, eficiência habitual do sono, distúrbios do sono, medicação para dormir e disfunção diurna. Cada um dos sete componentes é pontuado de
0 (sem dificuldades para o componente de interesse) a 3 (muito ruim). Esses escores dos componentes são combinados para gerar um escore PSQI global que reflete a qualidade do sono e os distúrbios durante um período de 1 mês. A pontuação global varia de 0 a 21, com pontuaçóes mais elevadas que indicam uma menor qualidade do sono ${ }^{37}$. Assim, o escore do PSQI foi utilizado para análise.

\section{Avaliação do medo de cair}

A FES-I é uma das escalas mais confiáveis para avaliar o medo de cair de indivíduos com $\mathrm{DP}^{38}$. Essa escala apresenta excelentes propriedades psicométricas e relatos de atividades básicas e complexas, tanto físicas quanto sociais. $\mathrm{O}$ indivíduo respondia o quanto ele estaria preocupado em cair se fizesse 16 atividades existentes na escala. As alternativas de respostas variam de um a quatro, sendo: 1) não estou preocupado, 2) um pouco preocupado, 3) moderadamente preocupado, e 4) muito preocupado. A pontuação da FES-I varia de 16 a 64, sendo que quanto maior o escore maior o medo de cair do indivíduo ${ }^{39}$. O escore da FES-I foi usado para análise estatística.

\section{Análise estatística}

Os resultados foram apresentados por meio de estatística descritiva padrão (média \pm desvio padrão). Os dados foram analisados quantitativamente quanto à normalidade (Shapiro-Wilk). Uma análise de regressão múltipla linear, o método forward stepwise foi empregado, e as variáveis independentes (UPDRS-III, TUG, BESTest, 1RM, MoCA, IDB, PSQI e FES-I) que apresentaram uma significante associação $(\mathrm{P}<0,05)$ com a variável dependente (PDQ-39) entraram no modelo. Apenas uma variável independente (i.e., FES-I) entrou no modelo de regressão múltipla. $\mathrm{O}$ coeficiente de correlação de Pearson two-tailed foi empregado para verificar as correlaçóes entre as variáveis independentes (i.e., que entraram no modelo de regressão múltipla) com a variável dependente. Adicionalmente, uma matriz de correlação de Pearson foi empregada para verificar as associaçóes entre os escores dos oito domínios do PDQ-39 (mobilidade, atividades de vida diária, estado emocional, estigma social, apoio social, cognição, comunicação e desconforto físico) com a variável independente que foi preditora do PDQ-39. Por fim, o nível de significância adotado no presente estudo para todas as análises foi de $\mathrm{P} \leq 0,05$. O software SAS 9, $2^{\circledast}$ (Institute Inc., Cary, NC, USA) foi usado para todas as análises estatísticas. 


\section{Resultados}

\section{Amostra}

Noventa e um indivíduos com DP se voluntariaram para o estudo e assinaram o termo de consentimento livre e esclarecido. Trinta indivíduos com DP não atenderam aos critérios de inclusão (artrite significante e doença cardiovascular), 15 indivíduos tiveram problemas familiares que os impediram de participarem do estudo, um indivíduo apresentou dores nas costas, um indivíduo morreu e cinco não quiseram continuar no estudo. Portanto, a amostra final deste estudo foi composta por 39 indivíduos (FIGURA 1). As características demográficas, antropométricas e clínicas dos indivíduos estáo inseridas na TABELA 1.

FIGURA 1 - Fluxograma de recrutamento dos indivíduos com doença de Parkinson (DP).

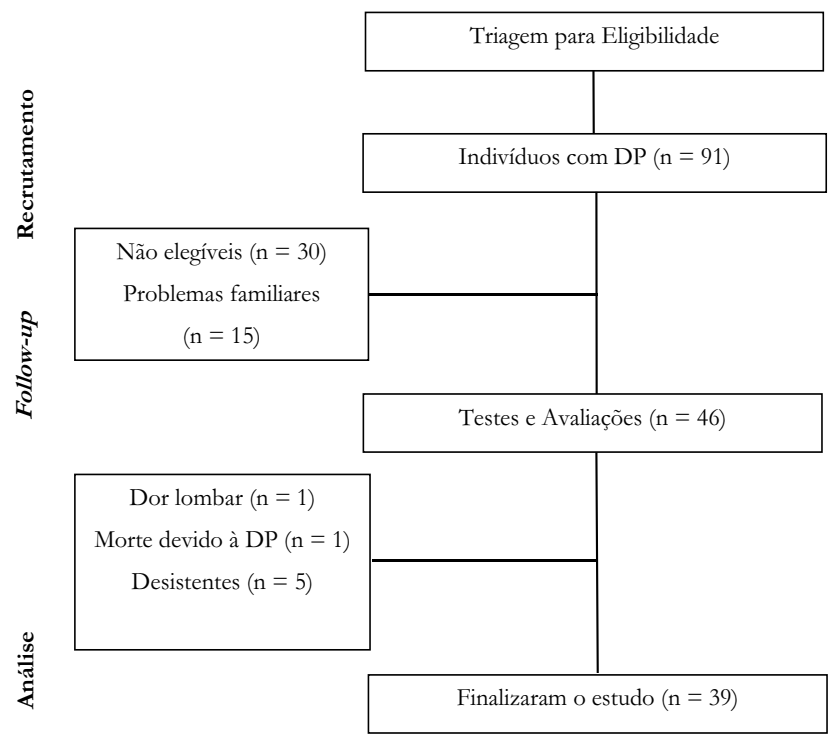

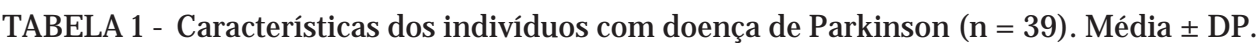

\begin{tabular}{lcc}
\hline Características & Média \pm DP & Range \\
\hline Demográfica & & \\
Homem/mulher (número) & $29 / 10$ & \\
Idade (anos) & $64,1 \pm 9,1$ & $50-80$ \\
Nível educacional (anos) & $8,3 \pm 2,6$ & $4-15$ \\
Antropométrica & & \\
Peso corporal (kg) & $70,5 \pm 9,8$ & $55-91$ \\
Altura (cm) & $1,7 \pm 0,1$ & $1,46-1,87$ \\
Índice de massa corporal (kg/m2) & $25,0 \pm 4,1$ & $18,2-35,2$ \\
Clínica & & \\
Mini Exame do Estado Mental (escore) & $28,6 \pm 1,7$ & $24-30$ \\
Tempo de diagnóstico da doença (anos) & $10,3 \pm 4,7$ & $4-20$ \\
Estágio na escala de Hoehn e Yahr (u.a) & $2,5 \pm 0,5$ & $2-3$ \\
UPDRS-III (escore) & $44,1 \pm 10,1$ & $19,5-60,2$ \\
Unidades equivalente de L-Dopa (mg•dia-1) & $568,7 \pm 270,0$ & $100-1150$ \\
\hline
\end{tabular}




\section{Regressáo múltipla Linear}

$\mathrm{Na}$ análise de regressão múltipla linear, utilizando o método forward stepwise, apenas o escore da FES-I entrou no modelo da regressão $(\mathrm{P}<0,05)$ e foi o principal preditor de qualidade de vida (PDQ-39) de indivíduos com DP (R2 ajustado $=0.73, \mathrm{P}<0,0001)$.

A FIGURA 2 apresenta o gráfico de correlação (coeficiente de correlação de Pearson) entre o escore da FES-I com o escore da PDQ-39 demonstrando uma associação significante, positiva e forte $(\mathrm{r}=$ $0,86 ; \mathrm{IC}=0,75$ a 0,$92 ; \mathrm{P}<0,0001)$.
A TABELA 2 apresenta os valores de correlação entre os escores dos oito domínios do questionário PDQ-39 com o escore da FES-I (única variável independente que entrou no modelo de regressão múltipla). Esta análise foi realizada no intuito de observar quais domínios poderiam apresentar associaçôes significantes com o escore do medo de cair. Interessantemente, todos os domínios apresentaram associaçôes significantes com o escore da FES-I (TABELA 2) demonstrando que o medo de cair pode ter uma possível interação com os domínios do PDQ-39.

FIGURA 2 - Coeficiente de correlação de Pearson two-tailed entre os escores do Questionário da Doença de Parkinson (PDQ-39) e da Escala Internacional de Eficácia de Quedas (FES-I) dos 39 indivíduos com doença de Parkinson.

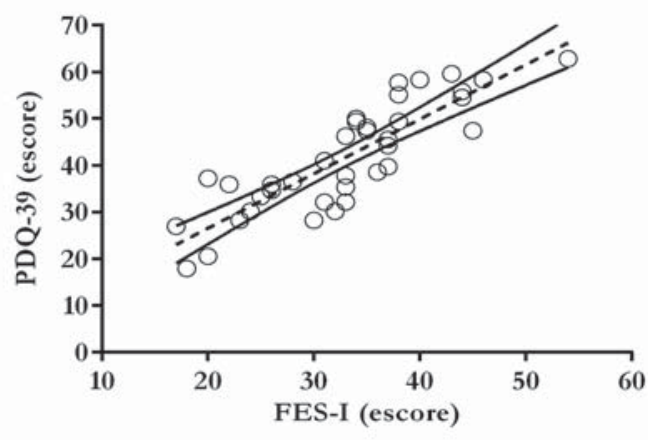

TABELA 2 - Resultados da matriz de correlação de Pearson entre os oito domínios do Questionário da Doença de Parkinson (PDQ-39) com a variável medo de cair dos indivíduos com doença de Parkinson $(\mathrm{n}=39)$.

\begin{tabular}{lcc}
\hline & \multicolumn{2}{c}{ FES-I } \\
\cline { 2 - 3 } Domínios do PDQ-39 & $\mathrm{r}$ & Valor de P \\
\hline Mobilidade (escore) & 0,48 & 0,002 \\
Atividades de vida diária (escore) & 0,64 & 0,001 \\
Estado emocional (escore) & 0,69 & 0,001 \\
Estigma social (escore) & 0,55 & 0,001 \\
Apoio social (escore) & 0,39 & 0,012 \\
Cognição (escore) & 0,59 & 0,001 \\
Comunicaçáa (escore) & 0,69 & 0,001 \\
Desconforto físico (escore) & 0,31 & 0,048 \\
\hline
\end{tabular}




\section{Discussão}

O presente estudo verificou dentre os sintomas motores, não motores e o medo de cair quais seriam os principais preditores de qualidade de vida em indivíduos nos estágios 2 e 3 da DP. O achado deste estudo mostrou que o medo de cair (FES-I) foi o único preditor de qualidade de vida dessa população, bem como todos os escores dos domínios do PDQ39 apresentaram associaçôes significantes com o escore da FES-I (TABELA 2). Assim, estes achados confirmam uma de nossas hipóteses.

Poucos estudos que compararam o impacto de diversas disfunçóes na qualidade vida de indivíduos com DP consideraram o medo de cair. Somente dois estudos encontrados na literatura analisaram o impacto do medo de cair na qualidade de vida dessa populaçãa ${ }^{40,41}$. Adicionalmente, vale ressaltar que os resultados desses estudos foram consistentes com os nossos, no entanto, foram utilizadas ferramentas indiretas para avaliar o medo de cair. Ainda, os autores utilizaram questionários genéricos para avaliação de déficits do equilíbrio que não avaliam especificamente o medo de cair, o que pode clinicamente confundir os resultados. Portanto, nosso estudo foi o primeiro a utilizar uma ferramenta válida, específica e de alta confiabilidade (FES-I) para avaliaçáa do medo de $\operatorname{cair}^{38}$ o que fortalece a generalização dos nossos resultados.

O medo de cair é altamente prevalente em indivíduos com $\mathrm{DP}^{9}, 10$ e um forte preditor de quedas e de restrição das atividades ${ }^{42}$, ${ }^{43}$. Isto ocasiona um ciclo vicioso que consequentemente impacta negativamente na qualidade de vida desses indivíduos ${ }^{9-11}$. É importante ressaltar que indivíduos com DP que relatam medo de cair também apresentam maior instabilidade postural ${ }^{9}$. Por exemplo, em nossa amostra os indivíduos com maiores escores da FES-I $(n=23)$ estavam no estágio 3 da DP (i.e., alguma instabilidade postural) e apresentavam os menores escores da BESTest. Assim, é possível sugerir que o medo de cair pode ser influenciado pelas alteraçóes no equilíbrio estático e dinâmico ocasionadas pela DP, consequentemente, déficits no equilíbrio podem ter um impacto negativo na qualidade de vida desses indivíduos. Ainda, escores da UPDRS-III >30 indicam início da presença de incapacidade, perda de autonomia e declínio da qualidade de vida $a^{44}$. De fato, $31(79,5 \%)$ indivíduos da nossa amostra apresentaram escores $>30$ demonstrando que a maioria dos indivíduos do presente estudo apresentavam uma qualidade de vida pobre. Assim, é possível sugerir que o medo de cair pode ser influenciado pelas alteraçóes motoras ocasionadas pela DP, consequentemente, déficits no equilíbrio e sintomas motores, podem impactar negativamente no medo de cair e consequentemente na qualidade de vida desses indivíduos.

A força do nosso estudo está primeiramente na utilização de ferramentas de mensuração válidas, fidedignas e sensíveis o suficiente para detectar e avaliar as disfunçóes propostas. Segundo, nossa amostra estava sob terapia medicamentosa estável, o que deixa os resultados do estudo mais consistentes. A principal limitação do nosso estudo é não ter avaliado os indivíduos no estado "off" da medição antiparkinsoniana o que não reflete verdadeiramente o estado real da doença, mas por outro lado, reflete o estado típico do paciente durante o dia.

Portanto, dentre as disfunçóes ocasionadas pela DP, o medo de cair é o principal preditor de qualidade de vida de indivíduos com moderada DP. Sendo assim, nossos resultados implicam no fato de que o medo de cair deve ser um alvo terapêutico para o tratamento da DP. No entanto, como este é um estudo transversal de associaçáo, nossos dados não podem estabelecer uma relação de causa e efeito entre essas variáveis.

\section{Agradecimentos}

Os autores agradecem aos voluntários da Associação Brasil Parkinson por participarem desta pesquisa e à Fundação de Amparo à Pesquisa do Estado de Sáo Paulo (FAPESP 2011/042423, 2012/03056-4 e 2013/04970-4) pelo financiamento deste projeto. 


\begin{abstract}
Predictor of quality of life in individuals with moderate Parkinson's disease

The purpose of this study was to verify among the motor and nonmotor dysfunctions and the fear of falling the main predictors for poor quality of life in individuals in stages 2 and 3 of Parkinson's disease (PD). Thirty-nine individuals with PD (64.1 \pm 9.1 years, $10.3 \pm 4.7$ duration of disease) evaluated in "on" state (fully medicated) participated of the study. Motor dysfunctions were assessed using the following tests: Unified Parkinson's Disease Rating Scale part III motor subscale score (UPDRS-III), timed up and go test, Balance Evaluation System Test (BESTest), one-repetition maximum (1RM) in the leg press. Nomotor dysfunctions were assessed using the following tests: Montreal Cognitive Assessment (MoCA), Beck Depression Inventory (BDI) and Pittsburgh Sleep Quality Index (PSOI). The fear of falling was assessed by Falls Efficacy Scale-International (FES-I). Quality of life was assessed using the Parkinson's Disease Questionnaire- 39 (PDQ-39). A multiple regression linear, stepwise method was employed. Only the FES-I score entered into multiple linear regression model and showed a high ability to explain the quality of life score of individual with PD (R2 adjusted $=0.73, p<0.0001$ ). Thus, as clinical implication, it is possible to suggest that physical training strategies that promote a decrease in fear of falling can positively impact on the quality of life of individuals with PD.
\end{abstract}

KEYwORDs: Fear of falling; Motor signs; Postural instability; Activities of daily living.

\title{
Referências
}

1. Fahn S. Description of Parkinson's disease as a clinical syndrome. Ann N Y Acad Sci. 2003;991:1-14.

2. Jankovic J. Parkinson's disease: clinical features and diagnosis. J Neurol Neurosurg Psychiatry. 2008;79(4):368-76.

3. Marsden CD. Parkinson's disease. Lancet. 1990;335(8695):948-52.

4. Muslimovic D, Post B, Speelman JD, Schmand B, de Haan RJ, Group CS. Determinants of disability and quality of life in mild to moderate Parkinson disease. Neurology. 2008;70(23):2241-7.

5. Barbieri FA, Rinaldi NM, Santos PC, Lirani-Silva E, Vitorio R, Teixeira-Arroyo C, et al. Functional capacity of Brazilian patients with Parkinson's disease (PD): relationship between clinical characteristics and disease severity. Arch Gerontol Geriatr. 2012;54(2):e83-8.

6. Carod-Artal FJ, Ziomkowski S, Mourao Mesquita H, Martinez-Martin P. Anxiety and depression: main determinants of health-related quality of life in Brazilian patients with Parkinson's disease. Parkinsonism Relat Disord. 2008;14(2):102-8.

7. Winter Y, von Campenhausen S, Arend M, Longo K, Boetzel K, Eggert K, et al. Health-related quality of life and its determinants in Parkinson's disease: results of an Italian cohort study. Parkinsonism Relat Disord. 2011;17(4):265-9.

8. Martinez-Martin P, Rodriguez-Blazquez C, Kurtis MM, Chaudhuri KR, Group NV. The impact of non-motor symptoms on health-related quality of life of patients with Parkinson's disease. Mov Disord. 2011;26(3):399-406.

9. Adkin AL, Frank JS, Jog MS. Fear of falling and postural control in Parkinson's disease. Mov Disord. 2003;18(5):496502 .

10. Bloem BR, Grimbergen YA, Cramer M, Willemsen M, Zwinderman AH. Prospective assessment of falls in Parkinson's disease. J Neurol. 2001;248(11):950-8.

11. Bloem BR, van Vugt JP, Beckley DJ. Postural instability and falls in Parkinson's disease. Adv Neurol. 2001;87:209-23.

12. Gazibara T, Pekmezovic T, Kisic Tepavcevic D, Svetel M, Tomic A, Stankovic I, et al. Health-related quality of life in patients with Parkinson's disease: Implications for falling. Parkinsonism Relat Disord. 2015;21(6):573-6.

13. Kadastik-Eerme L, Rosenthal M, Paju T, Muldmaa M, Taba P. Health-related quality of life in Parkinson's disease: a cross-sectional study focusing on non-motor symptoms. Health Quality Life Outcomes. 2015;13:83.

14. Menon B, Nayar R, Kumar S, Cherkil S, Venkatachalam A, Surendran K, et al. Parkinson's Disease, Depression, and Quality-of-Life. Indian J Psychol Med. 2015;37(2):144-8.

15. Hoehn MM, Yahr MD. Parkinsonism: onset, progression and mortality. Neurology. 1967;17(5):427-42.

16. Nantel J, McDonald JC, Bronte-Stewart H. Effect of medication and STN-DBS on postural control in subjects with 
Parkinson's disease. Parkinsonism Relat Disord. 2012;18(3):285-9.

17. Fasano A, Aquino CC, Krauss JK, Honey CR, Bloem BR. Axial disability and deep brain stimulation in patients with Parkinson disease. Nature Rev Neurol. 2015;11(2):98-110.

18. Visser JE, Allum JH, Carpenter MG, Esselink RA, Speelman JD, Borm GF, et al. Subthalamic nucleus stimulation and levodopa-resistant postural instability in Parkinson's disease. J Neurol. 2008;255(2):205-10.

19. Hughes AJ, Daniel SE, Kilford L, Lees AJ. Accuracy of clinical diagnosis of idiopathic Parkinson's disease: a clinico-pathological study of 100 cases. J Neurol Neurosurg Psychiatry. 1992;55(3):181-4.

20. Goetz CG, Poewe W, Rascol O, Sampaio C, Stebbins GT, Counsell C, et al. Movement Disorder Society Task Force report on the Hoehn and Yahr staging scale: status and recommendations. Mov Disord. 2004;19(9):1020-8.

21. Folstein MF, Folstein SE, McHugh PR. "Mini-mental state". A practical method for grading the cognitive state of patients for the clinician. J Psychiatr Res. 1975;12(3):189-98.

22. Corcos DM, Robichaud JA, David FJ, Leurgans SE, Vaillancourt DE, Poon C, et al. A two-year randomized controlled trial of progressive resistance exercise for Parkinson's disease. Mov Disord. 2013;28(9):1230-40.

23. Silva-Batista C, Corcos DM, Roschel H, Kanegusuku H, Gobbi LT, Piemonte ME, et al. Resistance training with instability for patients with Parkinson's Disease. Med Sci Sports Exerc. 2016;48(9):1678-87.

24. Dibble LE, Hale TF, Marcus RL, Gerber JP, LaStayo PC. High intensity eccentric resistance training decreases bradykinesia and improves Quality Of Life in persons with Parkinson's disease: a preliminary study. Parkinsonism Relat Disord. 2009;15(10):752-7.

25. Peto V, Jenkinson C, Fitzpatrick R. PDQ-39: a review of the development, validation and application of a Parkinson's disease quality of life questionnaire and its associated measures. J Neurol. 1998;245(Suppl 1):S10-4.

26. Fahn S, Elton RL, Members. UP. Unified Parkinson’s disease rating scale. In: Fahn S, Marsden CD, Goldstein M, Calne DB, editors. Recent developments in Parkinson's disease, Vol 2 Florham Park, NJ: Macmillan Healthcare Information. 1987:153-63, 293-304.

27. Berg KO, Maki BE, Williams JI, Holliday PJ, Wood-Dauphinee SL. Clinical and laboratory measures of postural balance in an elderly population. Arch Phys Med Rehabil. 1992;73(11):1073-80.

28. Podsiadlo D, Richardson S. The timed "Up \& Go": a test of basic functional mobility for frail elderly persons. J Am Geriatr Soc. 1991;39(2):142-8.

29. Rinalduzzi S, Trompetto C, Marinelli L, Alibardi A, Missori P, Fattapposta F, et al. Balance dysfunction in Parkinson's disease. BioMed research international. 2015;2015:434683.

30. Horak FB, Wrisley DM, Frank J. The Balance Evaluation Systems Test (BESTest) to differentiate balance deficits. Phys Ther. 2009;89(5):484-98.

31. Brown LE, Weir JP. ASEP procedures recommendation I: accurate assessment of muscular strength and power. J Exerc Physiologyonline. 2001;4(3):1-21.

32. Nasreddine ZS, Phillips NA, Bedirian V, Charbonneau S, Whitehead V, Collin I, et al. The Montreal Cognitive Assessment, MoCA: a brief screening tool for mild cognitive impairment. J Am Geriatr Soc. 2005 Apr;53(4):695-9.

33. Beck AT, Epstein N, Brown G, Steer RA. An inventory for measuring clinical anxiety: psychometric properties. J Consulting Clin Psychol. 1988 Dec;56(6):893-7.

34. Beck AT, Ward CH, Mendelson M, Mock J, Erbaugh J. An inventory for measuring depression. Archi Genl Psychiatry. $1961 ; 4: 561-71$.

35. Hogl B, Arnulf I, Comella C, Ferreira J, Iranzo A, Tilley B, et al. Scales to assess sleep impairment in Parkinson's disease: critique and recommendations. Mov Disord. 2010;25(16):2704-16.

36. Carpenter JS, Andrykowski MA. Psychometric evaluation of the Pittsburgh Sleep Quality Index. J Psychosomatic Res. 1998;45(1):5-13.

37. Buysse DJ, Reynolds CF, 3rd, Monk TH, Berman SR, Kupfer DJ. The Pittsburgh Sleep Quality Index: a new instrument for psychiatric practice and research. Psychiatry Res. 1989;28(2):193-213.

38. Jonasson SB, Nilsson MH, Lexell J. Psychometric properties of four fear of falling rating scales in people with Parkinson's disease. BMC Geriatr. 2014;14:66.

39. Yardley L, Beyer N, Hauer K, Kempen G, Piot-Ziegler C, Todd C. Development and initial validation of the Falls Efficacy Scale-International (FES-I). Age Ageing. 2005;34(6):614-9.

40. Brozova H, Stochl J, Roth J, Ruzicka E. Fear of falling has greater influence than other aspects of gait disorders on quality of life in patients with Parkinson's disease. Neuro Endocrinol Letters. 2009;30(4):453-7.

41. Grimbergen YA, Schrag A, Mazibrada G, Borm GF, Bloem BR. Impact of falls and fear of falling on health-related 
quality of life in patients with Parkinson's disease. J Parkinson's Disease. 2013;3(3):409-13.

42. Mak MK, Pang MY, Mok V. Gait difficulty, postural instability, and muscle weakness are associated with fear of falling in people with Parkinson's disease. Parkinsons Dis. 2012;2012:901721.

43. Mak MK, Pang MY. Fear of falling is independently associated with recurrent falls in patients with Parkinson's disease: a 1-year prospective study. J Neurol. 2009;256(10):1689-95.

44. Shulman LM, Gruber-Baldini AL, Anderson KE, Vaughan CG, Reich SG, Fishman PS, et al. The evolution of disability in Parkinson disease. Mov Disord. 2008;23(6):790-6.

\begin{tabular}{r|l} 
ENDEREço & \\
Carla Silva-Batista & \\
Escola de Educacaão Física e Esporte & Submetido: 19/05/2017 \\
Universidade de São Paulo & Revisado: 17/ 11/2018 \\
Av. Prof. Mello Moraes, 65 - Cidade Universitária & Aceito: 22/ 08/2018 \\
05508-030 - São Paulo - SP - Brasil & \\
E-mail: csilvabatista@usp.br & \\
&
\end{tabular}

24 • Rev Bras Educ Fís Esporte, (São Paulo) 2021J an-Mar;35(1):15-24 